\title{
Time discrimination in a monotonic, isochronous sequence
}

\author{
Anders Friberg ${ }^{\text {a) }}$ and Johan Sundberg \\ Royal Institute of Technology, Department of Speech Communication and Music Acoustics, Box 700 14, \\ S-100 44 Stockholm, Sweden
}

(Received 17 February 1995; revised 17 April 1995; accepted 28 June 1995)

\begin{abstract}
In acoustic communication timing seems to be an exceedingly important aspect. The just noticeable difference (jnd) for small perturbations of an isochronous sequence of sounds is particularly important in music, in which such sequences frequently occur. This article reviews the literature in the area and presents an experiment designed to resolve some conflicting results in the literature regarding the tempo dependence for quick tempi and relevance of music experience. The jnd for a perturbation of the timing of a tone appearing in an isochronous sequence was examined by the method of adjustment. Thirty listeners of varied musical background were asked to adjust the position of the fourth tone in a sequence of six, such that they heard the sequence as perfectly isochronous. The tones were presented at a constant interonset time that was varied between 100 and $1000 \mathrm{~ms}$. The absolute jnd was found to be approximately constant at $6 \mathrm{~ms}$ for tone interonset intervals shorter than about $240 \mathrm{~ms}$ and the relative jnd constant at $2.5 \%$ of the tone interonsets above $240 \mathrm{~ms}$. Subjects' musical training did not affect these values. Comparison with previous work showed that a constant absolute jnd below $250 \mathrm{~ms}$ and constant relative jnd above $250 \mathrm{~ms}$ tend to appear regardless of the perturbation type, at least if the sequence is relatively short. (C) 1995 Acoustical Society of America.
\end{abstract}

PACS numbers: 43.66.Mk, 43.75.Cd

\section{INTRODUCTION}

The duration of tones in a music performance typically deviates from their nominal values given by the score. These deviations are an important part of musical expressivity. The relevance of tone durations to music performance has been demonstrated in many previous investigations (Shaffer, 1981; Bengtsson and Gabrielsson, 1983; Clarke, 1982; Sloboda, 1983; Sundberg et al., 1991b; Todd, 1985; Repp, 1992a). However, the scientific interest has focused mainly on the analysis of music performances, while the perceptual aspect has been studied less intensively.

An interesting perceptual aspect is the just noticeable difference (jnd) in time in various situations. For example, what is the smallest noticeable quantity by which the onset of one or several tones can be displaced in time in a performance? Recently this question has been approached from a top-down, music performance point of view, by using real music examples in various ways (Repp, 1992b, 1994; Clarke 1989; Sundberg et al., 1991a; Friberg and Sundberg, 1994). Psychoacoustic approaches have also been tried in which the traditional single-interval or single-duration discrimination measurements have been extended to several time intervals.

The extension from a single-interval to several equidistant, consecutive intervals seems to be a natural development in studying time perception in a slightly more complex and musically relevant context; for example, a drummer produces quasi-isochronous sequences and obviously is required to "keep time" with an accuracy high enough so as to produce only imperceptible errors. The involvement of isochronous sequences adds however many degrees of freedom. In

${ }^{a)}$ E-mail: andersf@ speech.kth.se an isochronous sequence the following perturbations seem particularly important as illustrated in Fig. 1 (Schulze, 1978): (1) displacement of one time marker; (2) lengthening/ shortening of one interval; (3) cyclic displacement of, e.g., every second or every fourth time marker; and (4) tempo change; i.e., all interonset intervals (IOIs) are changed after a certain time marker in the sequence, or two sequences, each having different IOIs, are compared.

In addition, the position of the deviation, the length of the sequence, and the pitch of the time marker as a function of position can be varied in all applicable cases. Various aspects of the different perturbation types will be elucidated in the following review of the literature on isochronous sequences. The jnd values are in the following presented either in terms of absolute jnd, that is, in $\mathrm{ms}$, or in terms of relative jnd, that is, in percent of the IOI.

\section{PREVIOUS RESEARCH ON ISOCHRONOUS SEQUENCES}

\section{A. jnd for displacement and lengthening/shortening}

A number of investigations have analyzed perceptual phenomena associated with early or late arrival of one element within an isochronous sequence. As illustrated in Fig. 1 , two different situations may occur. One is that one of the IOIs is shortened or lengthened, but the arrival times of the subsequent time markers remain unchanged by a corresponding lengthening and shortening, respectively, of the subsequent IOI (type 1 in Fig. 1). In the other situation one of the IOIs is shortened or lengthened, but in this case all following time markers arrive correspondingly early or late (type 2 in Fig. 1).

Hibi (1983) measured discrimination threshold of for- 
1. Displacement

\

2. Lengthening/shortening

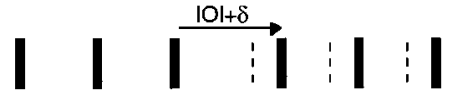

3. Cyclic displacement

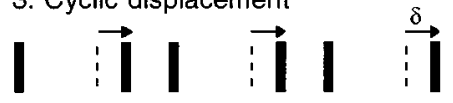

4. Tempo change

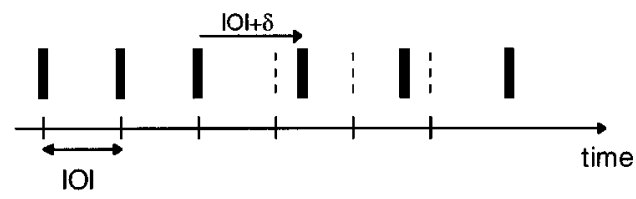

FIG. 1. Examples of different perturbation types in an isochronous sequence: 1, forward (late) displacement of one time marker with a small perturbation $\delta$; 2, lengthening of one interval; 3, cyclic displacement of every other time marker; and 4 , tempo difference where each interval after a certain point is lengthened with $\delta$.

ward and backward displacements of a single time marker (late and early arrival), and lengthening and shortening of a single IOI in a sequence of 15 tone bursts. The position of the perturbation occurred at three different locations in the middle of the sequence. The task of the three subjects was to report disturbances of the metrical regularity. The method of constant stimuli was used to estimate the $50 \%$ detection level. As can be seen in Fig. 2(a) two areas of constant relative jnd was found: $\mathrm{IOI}=400$ to $1000 \mathrm{~ms}$ and $\mathrm{IOI}=143$ to 250. For IOI $=400$ to $1000 \mathrm{~ms}$, the jnd was about $6 \%$ in all conditions, whereas for IOI $=143$ to 250 , the jnd value varied between $7.5 \%$ and $9 \%$ depending on the type of perturbation. In the transition area (IOI=250 to $400 \mathrm{~ms}$ ) the dependence on the type of perturbation was even higher.

Hirsh et al. (1990) measured the discrimination threshold for a forward displacement of a single time marker in sequences of 6 or 10 tones for $\mathrm{IOI}=50,100$, and $200 \mathrm{~ms}$. All tones were $1000-\mathrm{Hz}$ sinusoids with a duration of $20 \mathrm{~ms}$. They used a cued adaptive two-alternatives-forced-choice (2AFC) procedure where the amount of displacement was halved when subjects gave two consecutive correct answers and doubled for each incorrect answer. Contrary to Hibi, the absolute jnd was found to be constant, at least for $\mathrm{IOI}=100$ and $200 \mathrm{~ms}$. For IOIs $100 \mathrm{~ms}$ and longer, the absolute jnd was found to be independent of the position of the tone within the sequence. The average jnd over all positions and both sequence lengths are shown in Fig. 2(a) (IOI=100 and $200 \mathrm{~ms}$ ).

A slightly different type of perturbation was considered by Halpern and Darwin (1982). They measured the displacement discrimination of the last click of an isochronous sequence of four. The method of constant stimuli was used to determine the jnd for eight IOIs ranging from 400 to 1450 $\mathrm{ms}$. Converting the results to relative values showed that the relative jnd, expressed as the $50 \%$ detection level, was almost constant $(3.1 \%$ to $4.4 \%)$ over the whole IOI range analyzed [see Fig. 2(a)]. (a) Displacement and lengthening/shortening

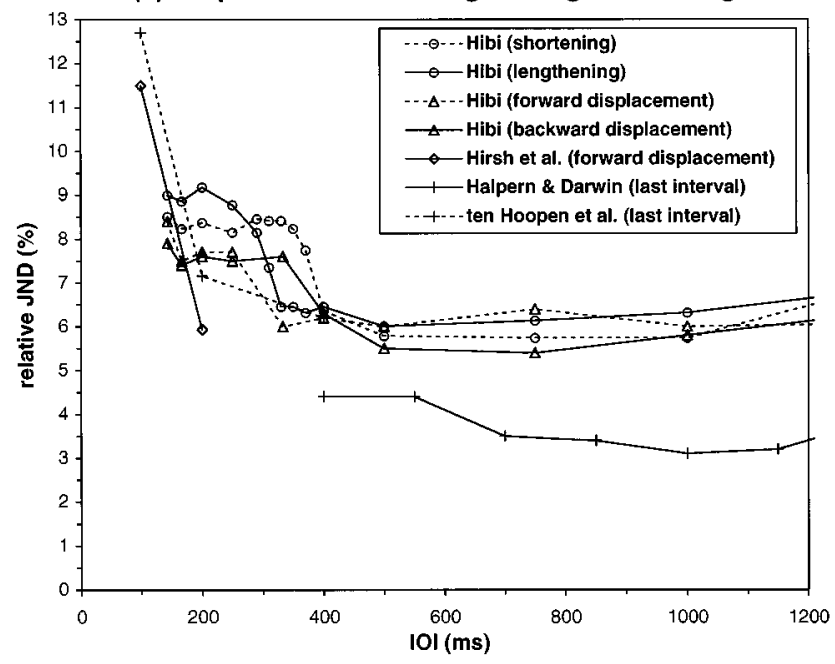

(b) Cyclic displacement

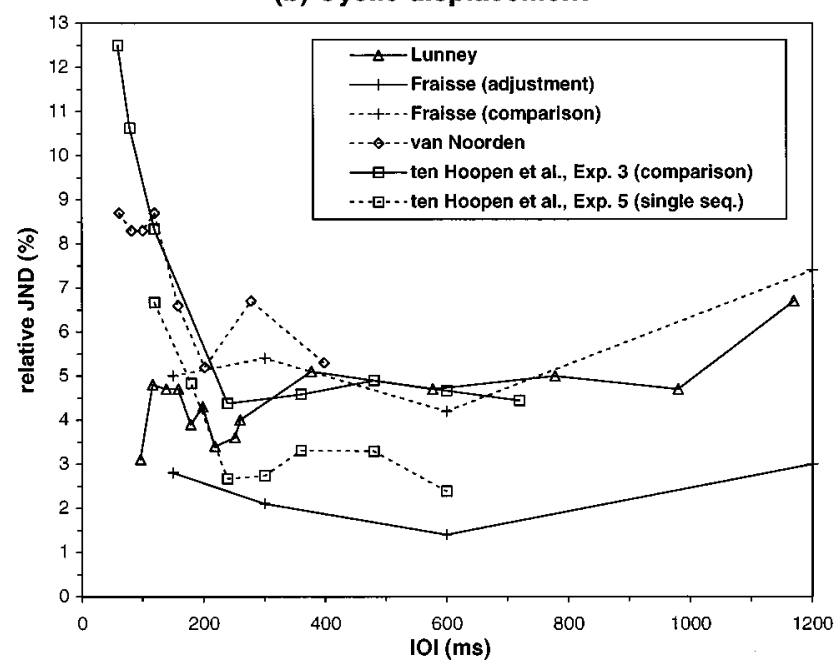

(c) Tempo

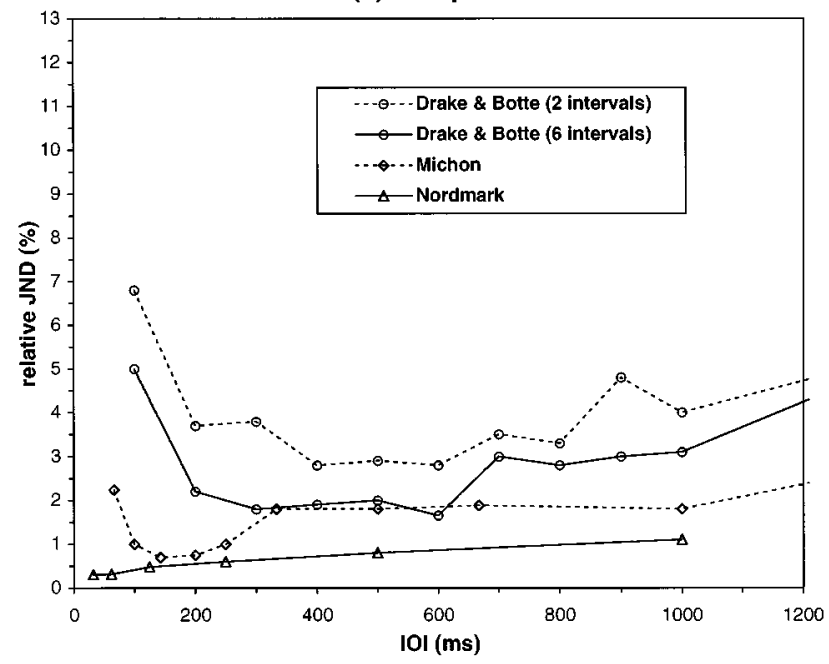

FIG. 2. An overview of time discrimination in an isochronous sequence for (a) displacement of one note or one time interval shortened or lengthened, (b) cyclic displacement, and (c) tempo.

The discrimination of a displacement of the final time marker in a sequence was also carefully investigated by ten Hoopen et al. (1995). They varied the number of intervals from 2 to 4 , and presented the stimuli at faster tempi 
$(\mathrm{IOI}=50$ to $400 \mathrm{~ms}$ ) than those examined by Halpern and Darwin. The time markers were 10-ms-long square waves with a frequency of $1000 \mathrm{~Hz}$. A method of constant stimuli was used and the jnd was estimated from the fitted cumulative normal distribution. There was no trend, as the number of intervals increased. The average absolute jnd was found to be constant at about $14 \mathrm{~ms}$ for $\mathrm{IOI}=100$ to $200 \mathrm{~ms}$ and $25 \mathrm{~ms}$ for $\mathrm{IOI}=400 \mathrm{~ms}$ [see Fig. 2(a)].

\section{B. jnd for cyclic displacement}

A common principle in music performance is to lengthen tones in stressed positions at the expense of the subsequent tone in unstressed position. This principle is applied both in Baroque music (Inégales) and in jazz (Swingfeel). In other types of music, e.g., Vienna waltz, other types of reiterant perturbations have been found (Bengtsson and Gabrielsson, 1983). A group of investigations have focused on such reiterant perturbations occurring in a sequence of time markers.

Lunney (1974) reported on a rather informal experiment in which he had used himself as the sole subject. He gradually displaced every fourth note in a continuous sequence until he could notice a metrical irregularity. IOI was varied from 30 to $3200 \mathrm{~ms}$. Although unfortunately, he did not report averages over repeated runs, his results showed an approximate JND of $5 \%$ for $350 \mathrm{~ms}<\mathrm{IOI}<1000 \mathrm{~ms}$ and a tendency to decrease for shorter IOIs [see Fig. 2(b)]. These results were reached after several weeks of practice.

A constant relative jnd for shorter IOIs was also found by Fraisse (1967) using a continuous alternating series where every other note was displaced. Both the method of adjustment and the method of constant stimuli were used, see Fig. 2(b). Five different tempi (IOI=150, 300, 600, 1200 and $2400 \mathrm{~ms}$ ) and four subjects were used in each test. In the adjustment task, the distance from the $25 \%$ and $75 \%$ interquantile points was taken as the measure of the jnd. Both methods showed a greater sensitivity at $\mathrm{IOI}=600 \mathrm{~ms}$ than at $\mathrm{IOI}=150,300$, and $1200 \mathrm{~ms}$ and the values were at least twice as large for the method of constant stimuli.

Also van Noorden (1975, p. 56) used two alternating, continuous sequences, but varied also the pitch. He used a tracking method similar to the one used by von Békésy for the determination of the auditory threshold. When all tones had the same pitch the relative jnd was about $8.5 \%$ for $\mathrm{IOI}=62$ to $120 \mathrm{~ms}$ and $6 \%$ for $\mathrm{IOI}=200$ to $400 \mathrm{~ms}$. For $\mathrm{IOI}=120$ to $200 \mathrm{~ms}$, the absolute jnd was approximately constant [see Fig. 2(b)].

ten Hoopen et al. (1994) made a series of experiments similar to that of van Noorden but with a larger range of IOI values and with finite rather than continuous sequences. The results were in accordance with van Noorden's. In experiment 3 , the absolute jnd was rather constant at $10 \mathrm{~ms}$ for $\mathrm{IOI}=60$ to $240 \mathrm{~ms}$ and the relative jnd was constant at about $4.5 \%$ for IOI $=240$ to $720 \mathrm{~ms}$ [see Fig. 2(b)]. They found that sequence length did not affect the results when the number of time markers was varied from 8 to 24 .

\section{C. jnd for tempo}

Michon (1964) measured the jnd for tempo using stepwise tempo changes, the so-called up-down staircase method. The subjects were trained and allowed to decide freely how long they wanted to listen to each tempo and could switch freely between the two tempi. Contrary to most other experimenters he found that the sensitivity increased for shorter IOI values. The maximum sensitivity was about $1 \%$ for IOIs between 100 and $200 \mathrm{~ms}$, and 2\% for IOIs between 300 and $1000 \mathrm{~ms}$ [see Fig. 2(c)].

Nordmark (1968) measured the frequency discrimination for the repetition of short pulses where the repetition rate varied from 1 to $4000 \mathrm{~Hz}$. In the infrafrequency range this is equivalent to measuring the jnd for tempo. He used the method of adjustment with an experimental setup very similar to Michon's. He found a close linear relationship between $\log$ IOI and $\log$ absolute jnd. For an IOI of $62.5 \mathrm{~ms}$ jnd was $0.2 \mathrm{~ms}$ and for $\mathrm{IOI}=1000 \mathrm{~ms}$ jnd was $11 \mathrm{~ms}$ [see Fig. 2(c)].

Drake and Botte (1993) measured the jnd for tempo when the number of presented intervals varied from 1 to 6 . An adaptive 2 AFC procedure converging on the $70.7 \%$ correct level was used. Isochronous sequences were presented in pairs in which the tempo differed slightly, and the subjects indicated which they perceived as the fastest. Drake and Botte found that the jnds for stimuli containing 2 to 6 intervals were considerably lower than the jnds for those containing one single interval. Contrary to the findings of Michon and Nordmark, the jnd increased for IOIs shorter than 400 ms. Overall, the sensitivity increased with the number of intervals in the sequence. The result for 2 and 6 intervals are shown in Fig. 2(c).

\section{General comments}

The various investigations summarized above demonstrate that for sequences of time intervals between tones of identical loudness, pitch, and timbre, the jnd for a perturbation in time is dependent upon a number of different experimental factors. Examples of such factors are interonset interval, number of time intervals included in the sequence, and type of perturbation. The number of relevant factors increase if musically more realistic stimuli are considered (see, e.g., Repp, 1992b).

The jnd values vary considerably. Some of this variation is due to methodological effects. Results obtained by an adjustment method are not directly comparable with the $50 \%$ detection level values obtained by, e.g., a forced-choice method; such a comparison is feasible after a doubling of the values obtained by the adjustment method (using the average standard deviation as the measure of the jnd). This approximate relationship has been confirmed theoretically (Cardozo, 1965), in pitch discrimination measurements (Wier et al., 1976), and in time discrimination measurements (Fraisse, 1967).

The investigations described above and illustrated in Fig. 2 cause several general observations and comments as follows.

(1) Our sensitivity to tempo change is clearly greater 
than our sensitivity to the other types of perturbations. This may be expected since a tempo change affects a greater number of intervals and consequently more information is available to the perception. Several mechanisms explaining this effect have been proposed (e.g., Drake and Botte, 1993). Since there are more cues in a cyclic displacement than in a single displacement one similarly would expect a higher sensitivity to cyclic displacement. In the results, only a slight trend to such a difference can be observed.

Schulze (1978), in a direct comparison, found tempo discrimination to be significantly better than displacement of one time marker or lengthening of a single interval (types 1 and 2 in Fig. 1). The detection of a lengthening of one interval was found to be slightly better (significant only in certain cases) than a displacement of one time marker.

(2) There is a middle IOI range of about 400 to $800 \mathrm{~ms}$ were the relative jnd is approximately constant. The jnd in that range is about $2 \%$ for tempo and between $4 \%$ and $6 \%$ for the other types of perturbation.

(3) Above $\mathrm{IOI}=800 \mathrm{~ms}$ some investigators have found that the jnd curves have a tendency to increase for longer IOIs.

(4) Below $\mathrm{IOI}=400 \mathrm{~ms}$ there is very little agreement between different measurements even for the same type of perturbation. The perception seems to change and a more complex situation is seen where the sensitivity in different experiments increase, decrease, or stay the same for shorter IOIs. A tendency for a constant absolute jnd below $\mathrm{IOI}=250$ ms, as proposed by ten Hoopen (1992) can be seen in some cases in all perturbation types. Interestingly, these are almost all experiments using short, finite length sequences. The only exception seems to be van Noorden who, by using continuous sequences, obtained a constant absolute jnd within the range of $\mathrm{IOI}=120$ to $200 \mathrm{~ms}$.

Many of the above measurements on the jnd exhibit a change of behavior for shorter IOIs. This may reflect two different perception mechanisms. This was proposed by Michon (1964) who found a breakpoint somewhere between 200 and $300 \mathrm{~ms}$. Hibi (1983) proposed that the perception of short IOIs (100-250 ms) is holistic, i.e., that the entire sequence is heard as a whole, and that the perception of the long IOIs (400-1000 ms) is analytic; i.e., the sequence is perceived interval by interval. He found the breakpoint at about $330 \mathrm{~ms}$.

Further support for the holistic/analytic perception model was obtained by Kohno (1992). He found that children under 3 to 4 years old could only tap in synchrony with a metronome at a fast IOI rate $(250 \mathrm{~ms})$ and not at slower rates (500 and $1000 \mathrm{~ms}$ ), thus suggesting that only one of the mechanisms were developed at that age. In another experiment he asked adult subjects to identify nonsense words produced with equal intersyllable intervals (ISI). However, before writing down the word on an answer sheet the subjects were required to perform a simple calculation task. The identification was significantly better for the words with short ISIs. This suggests that the words with short ISI $(250 \mathrm{~ms})$ could be remembered as a whole, whereas the words with longer ISIs, possibly processed event by event, was lost during the calculation task. ten Hoopen (1992) compared different sequential timing discrimination measurements. He observed that the $\mathrm{IOI}=250$ ms appeared to be a breakpoint for the perception, independently of the type of perturbation or the test method. He also found that for IOI=100 to $250 \mathrm{~ms}$ the absolute jnd was constant and proposed a pattern matching model for this range.

The scatter of the results from different experiments suggests the existence of various influential factors. The skill of discriminating small time deviations in isochronous sequences is similar to skills needed by musicians to produce high quality performances. Consequently, one would a priori assume that the subjects' musical experience would influence the sensitivity. Two of the above experiments considered the influence of musical experience. Halpern and Darwin (1982) in measuring displacement discrimination of the final tone in a sequence, did not find a higher sensitivity for musically trained subjects. The amount of formal music training varied from 0 to 12 years. On the other hand, Drake and Botte (1993) in measuring tempo discrimination found a significant effect of musical training.

\section{EXPERIMENT}

An experiment was carried out to further elucidate the as yet unclear dependence of the jnd for perturbation of type 1 especially at faster tempi, IOI $<400 \mathrm{~ms}$, which is a musically particularly important tempo range. The influence of musical training was also taken into account. Mainly to save subjects' time we chose an adjustment task where the discrimination of a displacement of one tone in an isochronous sequence of six tones of equal pitch was measured. All tones were separated by the same IOI except for the fourth one which could be moved back and forth in time relative to the other, fixed tones. We tried only one position for the variable tone; in a similar experiment, Hirsh et al. (1990) showed that there was no dependence of position within the interonset range we used in our experiment (100-1000 ms).

\section{A. Procedure}

A program written in Director Musices (Friberg, 1994), was used for running the entire test and recording the results. The subjects were initially asked to give some personal information regarding their musical experience: They were asked if they played any instrument, and in that case for how many years and how many hours per week. Then, they were presented with three practice trials with $\mathrm{IOI}=100,300$, and $500 \mathrm{~ms}$.

Thereafter, the test was run. It consisted of nine sequences with an IOI for the fixed tones of either 100, 130, 200, 240, 300, 380, 500, 800, or $1000 \mathrm{~ms}$. Each sequence appeared three times, so that the test contained a total of $3 \times 9=27$ stimulus presentations. The duration of the entire test ranged from 20 to 40 min depending on the subject. For each stimulus a scrollbar appeared on the computer screen. The subject could move the fourth note by adjusting the scrollbar with the mouse. As soon as it was moved, the six notes were played according to the new adjustment. The subjects were instructed to adjust the setting until they perceived the sequence as perfectly isochronous. There was no specific instruction as how to listen, such as to avoid mental subdi- 
vision of durations. The step size was $1 \%$. The displacements corresponding to the end positions on the scrollbar varied randomly between stimuli. The maximum positive displacement varied randomly from $35 \%$ to $65 \%$ and the maximum negative displacement varied from $-35 \%$ to $-65 \%$. The same was true for the initial displacement value that for each new stimulus was randomly varied from $-35 \%$ to $35 \%$. The program stored the displacement value, i.e., the discrepancy between the adjusted time position of the stimulus tone and its physical midpoint ( $\delta$, perturbation type 1 in Fig. 1). Also stored were the number of trials with the same stimulus as well as the largest displacements allowed by the scrollbar.

\section{B. Equipment}

The equipment consisted of a Macintosh II computer connected to a Yamaha FB01 synthesizer controlled via a MIDI (Loy, 1985) interface. A stimulus was chosen that resembled a real musical instrument played in a customary way. The sound used had a guitarlike timbre with a fast attack $(0.5 \mathrm{~ms})$ and an immediate exponential decay with a decay time of $0.5 \mathrm{~s}$ (when the amplitude is $1 / e$ of the initial value). When a new note was played, the previous note was terminated by a short final decay. This decay was set as short as possible without generating audible clicks. The pitch was constant at $262 \mathrm{~Hz}$ (C4). The stimuli were presented to the subjects over a loudspeaker placed in a damped studio. The volume was adjusted to a comfortable level.

In general, the precision associated with the MIDI standard is rather modest. Still, our experimental setup offered a timing accuracy of $\pm 2 \mathrm{~ms}$. This was due to the fact that a low number of MIDI events were transmitted simultaneously. The accuracy achieved was assumed to be sufficient in this case in view of the results from the earlier investigations mentioned above. This assumption was supported by the results from informal pretests.

\section{Subjects}

Most of the subjects were students of engineering at the Royal Institute of Technology, taking a course in music acoustics. A total of 29 students and one professional musician participated in the test. Of these, 23 reported that they were actively involved in music, with the number of years of musical activity ranging from 1-50.

\section{RESULTS}

For each IOI the mean and the standard deviation were calculated from each subjects' displacement values. The standard deviation was taken as the measure of the jnd. The mean value gives the constant error $(\mathrm{CE})$. It reflects the deviation of the point of subjective equality (PSE) from the point of objective equality (POE), that is, the difference from the physical midpoint. The average jnd values expressed in percent of the IOI (the Weber fraction) and in milliseconds, the constant error, and the number of repetitions are presented in Table I. Positive values of CE means that the subjects adjusted the position of the fourth tone so it came later than the physical midpoint. The relative jnd is also plotted in Fig. 3, where the dashes indicate $95 \%$ confidence intervals.
TABLE I. The average of the relative (in $\%$ of IOI) and absolute (in ms) jnd values, the constant error (CE) and number of repetitions for the displacement of the fourth tone in an isochronous sequence of six.

\begin{tabular}{lcccc}
\hline \hline IOI $(\mathrm{ms})$ & jnd $(\%)$ & jnd $(\mathrm{ms})$ & CE $(\%)$ & Repetitions \\
\hline 100 & 5.4 & 5.4 & -0.3 & 20.6 \\
130 & 4.8 & 6.2 & -1.2 & 16.6 \\
200 & 3.3 & 6.5 & -1.0 & 12 \\
240 & 2.5 & 6.0 & 0.1 & 11.8 \\
300 & 2.5 & 7.6 & -0.4 & 9.8 \\
380 & 2.7 & 10.1 & -0.6 & 8.3 \\
500 & 2.1 & 10.7 & -0.4 & 8.1 \\
800 & 2.7 & 21.4 & -0.6 & 7.4 \\
1000 & 2.6 & 26.5 & -0.1 & 8.8 \\
\hline \hline
\end{tabular}

For IOIs $=240 \mathrm{~ms}$ and longer the results are in accordance with Weber's law, that is, the relative jnd is constant, see Fig. 3; in average the jnd was $2.5 \%$ for $\mathrm{IOI}=240$ to 1000 $\mathrm{ms}$. For shorter IOIs (100 to $240 \mathrm{~ms}$ ) the absolute jnd is constant, in average $6 \mathrm{~ms}$.

To further confirm that the absolute and relative jnd were constant for short and long IOIs, respectively, three repeated measure ANOVAs were performed with the jnd as the dependent variable. In the first, the main effect of IOI on the relative jnd was checked and was found to be significant $(p<0.0001)$. In the second, a partial ANOVA was performed for IOI values from 240 to $1000 \mathrm{~ms}$. The main effect of IOI gave a nonsignificant result $(p<0.68)$ confirming that the relative jnd is constant in that range. In the third test a partial ANOVA was performed for the IOI values from 100 to 240 $\mathrm{ms}$, using the absolute jnd as the dependent variable. The main effect of IOI was not significant $(p<0.77)$ confirming that the absolute jnd was constant in that range.

Thus these results clearly supported the assumption of Hibi (1983) and others that two different processes are used for the perception of time intervals. Furthermore, the breakpoint at about $250 \mathrm{~ms}$ and the constant absolute jnd for IOIs between 100 and $250 \mathrm{~ms}$ confirms the findings by ten Hoopen (1992).

The subjects tended to scatter their adjustments around a

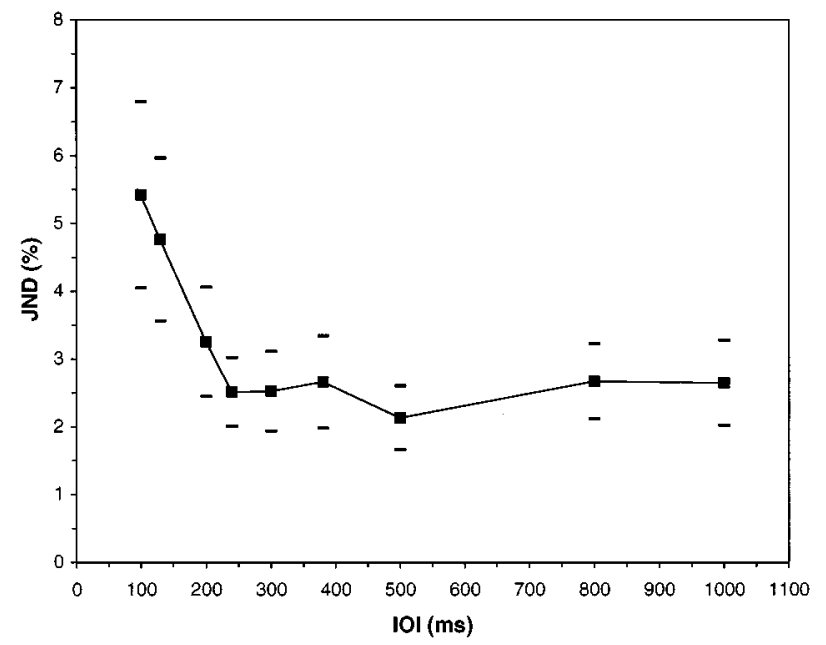

FIG. 3. The relative jnd as a function of interonset interval for the displacement of the fourth note in a six note sequence. The dashes indicate $95 \%$ confidence intervals for each value. 
Short sequences ( $<10$ intervals)

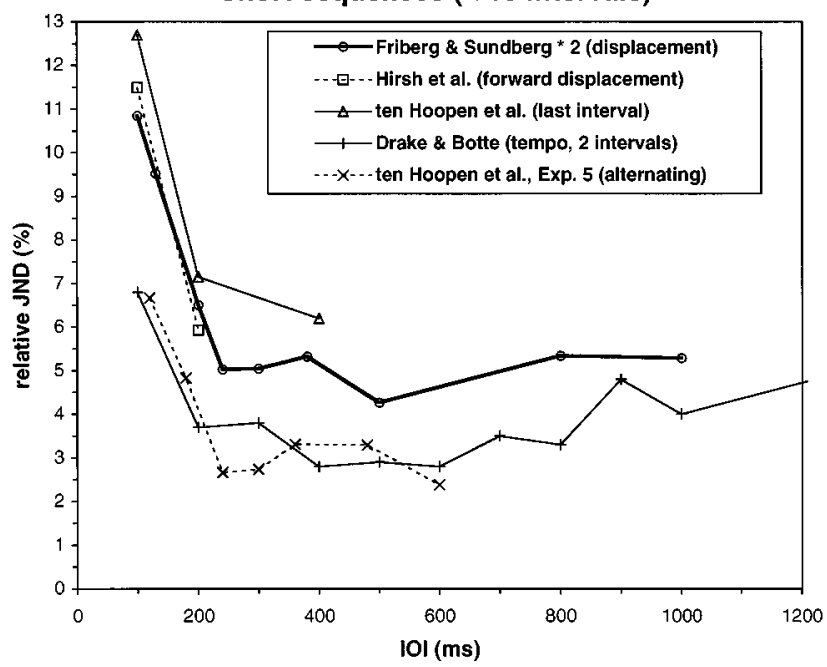

FIG. 4. Comparison with previous work. All measurements using isochronous sequences with less than ten intervals from Fig. 2 are shown together with the present result.

small negative deviation from the physical midpoint rather than around a zero deviation, as shown by the $\mathrm{CE}$ values in Table I. A $t$ test of CE averaged overall IOI values, indicated that the average $\mathrm{CE}$ was significantly less than zero (mean= $-0.5, p<0.0003)$. Interestingly, the only value that was positive was for $\mathrm{IOI}=240 \mathrm{~ms}$, i.e., at the breakpoint where the jnd curve change direction. A repeated measures ANOVA was performed to find out if the $\mathrm{CE}$ varied with the IOI value. The result was negative indicating no significant variation of the $\mathrm{CE}$ as a function of IOI $(p<0.5)$. As the deviations were small and for shorter IOIs less than the precision of the equipment, no further conclusions can be drawn.

Musical experience was not found to have any influence on the sensitivity. There was no significant correlation between musical experience and jnds averaged over all IOIs.

The number of times that each subject listened to the same stimulus may be taken as a measure of the difficulty of the task. In Table I, the average over all subjects of the number of repetitions are listed. Interestingly, the number of repetitions follows the same trend as the relative jnd. It is fairly constant for $\mathrm{IOI} \geqslant 380 \mathrm{~ms}$, and increases for shorter IOIs. This suggests that the perceived difficulty of the task depended on the relative jnd. Furthermore, there was no influence of musical experience in this regard.

There were no influence of training during the experiment. No significant correlation was found between each subject's jnd and number of repetitions, both averaged over all IOIs.

\section{DISCUSSION}

\section{A. Comparison with previous measurements}

Figure 4 compares our results with all of the previously mentioned investigations which used short isochronous sequences (number of intervals $<10$ ). To facilitate comparison our results have been multiplied by a factor of 2 , for reasons explained above. As can be seen in Fig. 4 all curves show the same overall trend irrespective of type of perturbation and method of measurement: a constant absolute jnd for IOI $=100$ to $250 \mathrm{~ms}$ and a constant relative jnd above $250 \mathrm{~ms}$.

The results of the Drake and Botte (1993) pertain to comparison of isochronous sequences consisting of two intervals (three tones). The shape of their curves is quite similar to ours with regard to the increase toward shorter time intervals and a relatively constant value for intermediate intervals. However, for longer time intervals their curves show an upward slope, which was not found in our results. This upward slope for longer intervals is also seen in the results by, e.g., Lunney (1974).

Hibi (1983) used the same type of perturbation as in the present investigation, but surprisingly his results diverge somewhat from ours. His discrimination results for backward and forward displacements were shown in Fig. 2(a). For IOI $>400 \mathrm{~ms}$ the relative jnd is constant, as in our investigation. However, even for short IOIs Hibi obtained an approximately constant relative jnd. This is not seen in any of the measurements shown in Fig. 4. Interestingly, Hibi used longer sequences of 15 time markers instead of the three to ten time markers that were used in the remaining measurements presented in Fig. 4. Most results from other investigations using longer sequences show a great variation and no trend to a constant absolute jnd for the IOI range 100 to 400 ms (Fraisse, 1967; Lunney, 1974; Michon, 1964; Nordmark, 1968). The only exception to this overall trend seems to be the results by van Noorden (1975) and ten Hoopen et al. (1994).

Summarizing, the length of the sequence seems to be more influential than the type of perturbation or the method used. The absolute jnd seems to be constant in the IOI range 100 to $250 \mathrm{~ms}$ at least when the sequence is relatively short.

\section{B. Range of constant absolute jnd}

The switch from a constant relative jnd to a constant absolute jnd occurs in the vicinity of $250 \mathrm{~ms}$. Thus, from the point of view of absolute duration sensitivity, is greatest for IOIs between 100 and $250 \mathrm{~ms}$, approximately. This is interesting from different aspects.

In musical performance short tones of durations in the range of 100 to $250 \mathrm{~ms}$ occur frequently, and lengthening of tone duration is often used for expressive purposes. Thus, from listening to music, we may be particularly trained to detect perturbations in this range of durations.

Durations of speech sounds in neutral speech seem to vary within a similar range (Klatt and Cooper, 1975; Crystal and House, 1988). Lengthening of syllables is an important cue for emphasis and other prosodic effects. Thus also from listening to speech we may be trained to detect perturbations at this magnitude of durations. Klatt and Cooper found jnd values for change of syllable duration in the range of 26 to $59 \mathrm{~ms}$. These rather large magnitudes may also reflect onset and context characteristics.

\section{Relevance of musical experience}

In the present experiment no positive effect of musical experience was found. The same results were obtained by 
Halpern and Darwin (1982) for the displacement discrimination of the last click in a sequence of four. On the other hand, Drake and Botte (1993) found a significant difference between musicians and nonmusicians with regard to tempo discrimination.

One reason for the lack of influence of musical experience could be that there was too little difference between our subject groups. For example, only one of our subjects was a professional musician. On the other hand, several subjects had quite extensive musical experience, and an analysis of the individual data revealed nothing that suggested a difference even for the most experienced subjects. Still, it is possible that a greater number of full-time professional musicians would have given a different result.

Another possible explanation is that there is a clear difference between listening and performance. Previously it has often been assumed that perception and performance of time are using the same mechanism. However, Sternberg et al. (1982) compared results based on perception and production of fractions of time intervals and found diverging results. This supports the hypothesis that the perception and performance of timing are different skills. Perhaps the skill to discriminate events of differing durations is acquired early or even prenatally, while musicians have to train themselves to perform a sufficiently accurate timing.

\section{Grouping and subdivision}

Musical phrases are often performed with a lengthening of the last note or with a ritardando in the end (see, e.g., Todd, 1985; Gabrielsson, 1987). This principle is mirrored in the detection ability; it is harder to detect a lengthening at the end of a phrase than in the middle of a phrase (Repp, 1992b, 1994). In short rhythmic patterns, Drake (1993) found similarly that the detection of a lengthening was dependent on position. Both these findings seem to support the hypothesis that lengthenings are harder to detect at group boundaries, both for longer phrases (Repp) and for shorter groups such as melodic gestures (Drake).

Even within an isochronous sequence mental grouping, i.e., subjective rhythmization, can occur. When we listen to an isochronous sequence, we tend to perceive chunks of tones, each of which consists of several tones (see, e.g., Parncutt, 1994). In our experiment, the subjects could manipulate the timing of the fourth note in a sequence of six. Such a sequence can be grouped in different ways, e.g., two groups of three, one group of four preceded by an upbeat and followed by a final tone, or one group of four followed by a group consisting of the two final tones. It is likely that the subjects chose different mental groupings, which may have affected the results. A predominance of the last mentioned patterning may be the reason why the PSE, averaged over all IOIs, occurred significantly earlier than the POE.

The effect of mental grouping might also explain some of the variance seen in previous investigations using longer sequences. If a greater number of time markers is presented, a greater number of grouping alternatives would a priori be available to the listener. For example, in tempo discrimination, it is possible to listen to groups of time markers and make the discrimination on the basis of the IOI for the groups instead of the individual IOIs. This would imply that the listener would be able to "transpose" the sensitivity in a certain tempo range to another tempo range! However, in a cyclic displacement the same processing may not be possible since the grouping is already enforced and the IOI for the groups is not affected. This may explain why jnd is not affected by sequence length in cyclic displacement (ten Hoopen et al., 1994).

For slow tempi some, but not all investigators have observed an increase of the jnd with increasing IOI. An explanation could be that our subjects could use mental subdivision for longer time intervals; the first three tones could be used to established a reference tempo inviting to a subdivision that was then carried over to the fourth tone. Mental subdivision of a sequence is probably not favored in the same manner in tempo discrimination experiments. It might be rewarding to control for grouping in future attempts to measure timing discrimination in isochronous sequences.

\section{ACKNOWLEDGMENTS}

The authors are indebted to Professor André-Pierre Benguerel, Canada for calling our attention to the work of S. Hibi. We gratefully acknowledge the many valuable suggestions from two anonymous reviewers, Bruno Repp, Gert ten Hoopen, Alf Gabrielsson, and Eric Prame. We also would like to thank the subjects for their participation. This work was supported by a grant from The Swedish Council for Research in the Humanities and Social Sciences (HSFR).

Bengtsson, I., and Gabrielsson, A. (1983). "Analysis and synthesis of musical rhythm," in Studies of Music Performance, edited by J. Sundberg (Royal Swedish Academy of Music, Stockholm), Publication No. 39, pp. 27-60.

Cardozo, B. L. (1965). "Adjusting the Method of Adjustment: SD vs DL," J. Acoust. Soc. Am. 37, 786-792.

Clarke, E. F. (1982). "Timing in the performance of Erik Satie's Vexations," Acta Psychol. 50, 1-19.

Clarke, E. F. (1989). "The perception of expressive timing in music," Psychol. Res. 51, 2-9.

Crystal, T. H., and House, A. S. (1988). "Segmental durations in connectedspeech signals: Current results,” J. Acoust. Soc. Am. 83, 1553-1573.

Drake, C. (1993). "Perceptual and performed accents in musical sequences," Bull. Psychonom. Soc. 31(2), 107-110.

Drake, C., and Botte, M-C. (1993). "Tempo sensitivity in auditory sequences: Evidence for a multiple-look model," Percept. Psychophys. 54, 277-286.

Fraisse, P. (1967). "Le seuil différentiel de durée dans une suite régulière d'intervalles," Année Psychol. 1, 43-49.

Friberg, A., and Sundberg, J. (1994). "Just noticable difference in duration, pitch and sound level in a musical context," in Proceedings of 3rd International Conference for Music Perception and Cognition, Liège, 1994, edited by I. Deliège (ESCOM, Liège), pp. 339-340.

Friberg, A. (1994). "Director Musices Demo 1.2," in Information Technology and Music, CD-ROM produced by the Royal Swedish Academy of Engineering Science.

Gabrielsson, A. (1987). "Once again: the theme from Mozart's piano sonata in A major (K. 331) A comparison of five performances," in Action and Perception in Rhythm and Music, edited by A. Gabrielsson (Royal Swedish Academy of Music, Stockholm), Publication No. 55, pp. 81-103.

Halpern, A. R., and Darwin, C. J. (1982). "Duration discrimination in a series of rhythmic events," Percept. Psychophys. 31(1), 86-89.

Hibi, S. (1983). "Rhythm perception in repetitive sound sequence," J. Acoust. Soc. Jpn. (E) 4, 83-95.

Hirsh, I. J., Monahan, C. B., Grant, K. W., and Singh, P. G. (1990). "Studies in auditory timing: 1. Simple patterns," Percept. Psychophys. 47(3), 215226. 
Klatt, D. H., and Cooper, W. E. (1975). "Perception of Segment Duration in Sentence Contexts," in Structure and Process in Speech Perception, edited by A. Cohen and S. G. Nootboom (Springer-Verlag, Berlin).

Kohno, M. (1992). "Two mechanisms of processing sound sequences," in Speech Perception, Production and Linguistic Structure, edited by Y. Tohkura et al. (Ohmsha, Tokyo and IOS, Amsterdam), pp. 287-293.

Loy, G. (1985). "Musicians make a standard: The MIDI phenomenon," Comput. Music J. 9(4), 8-26.

Lunney, H. W. M. (1974). "Time as heard in speech and music," Nature 249, 592.

Michon, J. A. (1964). "Studies on subjective duration. I. Differential sensitivity in the perception of repeated temporal intervals," Acta Psychol. 22, $441-450$

Nordmark, J. O. (1968). "Mechanisms of Frequency Discrimination," J. Acoust. Soc. Am. 44, 1533-1540.

Parncutt, R. (1994). "A Perceptual Model of Pulse Salience and Metrical Accent in Musical Rhythms," Music Percept. 11(4), 409-464.

Repp, B. K. (1992a). "Diversity and commonality in music performance: An analysis of timing microstructure in Schumann's Träumerie," J. Acoust. Soc. Am. 92, 2546-2568.

Repp, B. K. (1992b). "Probing the cognitive representation of musical time: Structural constraints on the perception of timing perturbations," Cognition 44, 241-281.

Repp, B. K. (1994). "Detectability of rhythmic perturbations in musical contexts: effects of metrical structure and of musical experience," in Proceedings of 3rd International Conference for Music Perception and Cognition, Liège, 1994, edited by I. Deliège (ESCOM, Liège), pp. 405-406.

Schulze, H.-H. (1978). "The detectability of local and global displacements in regular rhythmic patterns," Psychol. Res. 40, 173-181.

Shaffer, L. (1981). "Performances of Chopin, Bach and Bartok: studies in motor programming," Cog. Psychol. 13, 326-276.
Sloboda, J. A. (1983). "The communication of musical metre in piano performance,” Q. J. Exp. Psychol. 35, 377-396.

Sternberg, S., Knoll, R., L., and Zukofsky, P. (1982). "Timing by Skilled Musicians," in The Psychology of Music, edited by D. Deutsch (Academic, New York).

Sundberg, J., Friberg, A., and Frydén, L. (1991a). “Threshold and preference Quantities of Rules for Music Performance," Music Percept. 9(1), 71-92.

Sundberg, J., Friberg, A., and Frydén, L. (1991b). "Common Secrets of Musicians and Listeners-An analysis-by-synthesis Study of Musical Performance," in Representing Musical Structure, edited by P. Howell, R. West, and I. Cross (Academic, London).

ten Hoopen, G. (1992). "Temporal processing of fast auditory patterns," Paper presented at the 2nd International Conference on Music Perception and Cognition, Los Angeles.

ten Hoopen, G., Boelaarts, L., Gruisen, A., Apon, I., Donders, K., Mul, N., and Akerboom, S. (1994). "The detection of anisochrony in monaural and interaural sound sequences," Percept. Psychophys. 56(1), 110-120.

ten Hoopen, G., Hartsuiker, R., Sasaki, T., Nakajima, Y., Tanaka, M., and Tsumura, T. (1995). "Auditory isochrony: time-shrinking and temporal patterns," Perception 24, 577-593.

Todd, N. P. (1985). "A model of expressive timing in tonal music," Music Percept. 3, 33-58.

van Noorden, L. P. A. S. (1975). Temporal Coherence in the Perception of Tone Sequences (Institute for Perception Research, Eindhoven).

Wier, C. C., Jesteadt, W., and Green, D. M. (1976). "A comparison of method-of-adjustment and forced-choice procedures in frequency discrimination," Percept. Psychophys. 19(1), 75-79. 